\title{
Risk Assessment and Material Suitability Evaluation on Static Equipment of Hydrofluoric Acid Alkylation Unit
}

\author{
Weihua Wang, Duhui Lu, Xiang Li and Lin Liang *
}

Citation: Wang, W.; Lu, D.; Li, X.; Liang, L. Risk Assessment and Material Suitability Evaluation on Static Equipment of Hydrofluoric Acid Alkylation Unit. Processes 2021, 9, 1464. https://doi.org/10.3390/ pr9081464

Academic Editor: Sebastián Sánchez Villasclaras

Received: 26 July 2021

Accepted: 16 August 2021

Published: 21 August 2021

Publisher's Note: MDPI stays neutral with regard to jurisdictional claims in published maps and institutional affiliations.

Copyright: (c) 2021 by the authors. Licensee MDPI, Basel, Switzerland. This article is an open access article distributed under the terms and conditions of the Creative Commons Attribution (CC BY) license (https:// creativecommons.org/licenses/by/ $4.0 /)$.
Chinese Special Equipment Inspection and Research Institute, Beijing 100029, China; weihua0642@163.com (W.W.); luduhui@126.com (D.L.); lixiang@csei.org.cn (X.L.)

* Correspondence: abc13911808998@163.com; Tel.: +86-139-1180-8998

\begin{abstract}
In 2005, a $60 \mathrm{kt} / \mathrm{a}$ alkylation (ALK) unit began to resume production in the Second Oil Refinery Plant of Beijing Yanshan Petrochemical Company. There have been many leak cases from pipeline welds, valve body, flange, etc. After a half-year period of operation, production process is stable. However, the operation of the hydrofluoric (HF) acid ALK unit has been suffered from corrosion problems. There are no clear answers and references to the following problems. What types of corrosion are currently in the main equipment for HF acid ALK unit? What does cause equipment corrosion? What are the main influencing factors for corrosion? What measures can be taken to reduce the corrosion of HF acid? In this paper, considering the acid-related conditions of the ALK unit, the damage mechanism and damage rate analysis were carried out to calculate the safety risk of the static equipment of the ALK unit. Based on the damage mechanism and failure history, the material suitability of the ALK unit was investigated. The anti-corrosion measures and recommended materials for important corrosion parts of the ALK unit were proposed. It is meaningful for reducing the number of shutdowns of ALK units and maintaining safe and stable operation of the unit.
\end{abstract}

Keywords: risk assessment; material suitability evaluation; management strategy; failure possibility; failure consequence

\section{Introduction}

An alkylation (ALK) unit was set up in a Beijing petrochemical refining plant in 1988. Because of the poor Beijing petroleum and natural gas market at that time, the unit had not been used until 2005. With the increase of Beijing gasoline standard requirements, the alkylation unit was started to resume production. However, the operation of hydrofluoric (HF) acid ALK unit has been suffered from corrosion problems. The longest continuous operation time did not exceed 35 months. Three consecutive HF leakage accidents occurred in equipment of HF acid ALK units abroad in 2009. The risk assessment and material suitability evaluation of HF acid ALK units are good methods to extend the operating period of the unit, reduce injury of operators, and improve economic benefits of plants.

The risk assessment methods on equipment of plants have been widely used in China and abroad. Philippa Morre investigated an extending internal examination interval for pressure vessels using a Risk Based Inspection (RBI) approach [1]. Song Chunchoi implemented a Risk-Based Inspection for the refinery plant [2]. Wu Wenfang introduced a Risk-based inspection and maintenance in process plants and their practices in Taiwan [3]. Akanni Joseph performed a Risk Based Inspection (RBI) approach in asset integrity management of oil and gas facilities [4]. Hazard and operability analysis was focused on hydrocarbon alkylation units by Whittle, D.K. Because of the inherent hazards associated with the use of $\mathrm{HF}$ or $\mathrm{H}_{2} \mathrm{SO}_{4}$ as catalysts in the alkylation process, particular attention was focused on hazards involving potential releases of these toxic process materials [5]. Gary Beevers introduced a report about the Refinery Alkylation Research Action Project. To access the risk of an HF release from refineries, a 198 question survey was developed. The long-term solution is to replace HF alkylation with safer systems not requiring the 
use of so toxic a chemical. In the meantime, exiting alkylation units can and be made safer [6]. A two-phase jet model for predicting the HF rainout (capture) in HF/additive releases by R. Muralidhar [7]. Juliana Puello evaluated the hazards in the alkylation unit at a refinery located in Latin America, applying HAZOP, LOPA, and SIL methodologies [8]. In China, Wang Ruien et al. conducted risk assessment, inspection and verification on Catalytic Cracking Unit [9]. Yan Tingjun et al. introduced an application of RBI technology in delayed coking unit of refinery [10]. Wang Bin introduced an application of RBI in ethylene plant [11]. Shi Kai et al. conducted a risk analysis of pipeline system of natural gas installation based on RBI [12]. Liang Jiankuo carried out a RBI in pressure-bearing special equipment at LNG receiving stations [13]. Liu Wensuo performed a risk assessment of pressure vessel for methanol gasification unit based on RBI technology [14]. However, the RBI method has not been applied to the unit. The leakage and diffusion results under the largest credible accident have not been discussed. In this paper, Section 2 analyses the risk source of static equipment of ALK unit. Section 3 performs the risk assessment on static equipment of ALK unit. Risk assessment results, material suitability evaluation, and the management strategies of process anti-corrosion and equipment anti-corrosion were proposed in Section 4. Finally, Section 5 summarizes conclusions of this work.

\section{Risk Source Analysis of Static Equipment in HF Acid ALK Unit}

\subsection{Damage Driving Force}

HF acid mainly locates in reaction system, acid regeneration system, column top circulation system of fractionation tower and depropane tower, acid recovery system, butane defluorination system, and propane defluorination system in the HF acid ALK unit. Most of the equipment materials involved in the acid system are carbon steel and Monel alloy for the HF acid ALK unit. When it contacts with high concentration acid, for example water content less than $3 \%$, a stable iron fluoride layer is formed inside the equipment. It provides sufficient protection for carbon steel against further corrosion by HF acid. However, when the materials contact with an acid/water mixture, especially HF acid with a high water content, an unstable and unprotected hydrate scale is formed, which will cause further corrosion of the equipment. The corrosion rate will be greatly increased.

\subsection{Main Damage Parts and Main Corrosion Failure Modes}

\subsubsection{Main Damage Parts}

HF acid can react with most metals at room temperature to form metal fluorides and release hydrogen protons. HF acid reacts with Monel to form nickel fluoride. It adheres to the metal surface to form a dense protective layer, which can passivate the metal surface and protect the metal from further corrosion. However, when the environmental condition changes, the protective layer may be damaged, which will lead to an increased corrosion.

When metal components are in the medium, there is a particular small gap, usually $0.025 \mathrm{~mm}$ to $0.1 \mathrm{~mm}$, between the metal and metal or metal and non-metal. The width is sufficient to allow the medium to enter the gap. An accelerated metal corrosion happened in the gap because the liquid electrolyte is in a stagnant state. In the HF acid medium, crevice corrosion usually locates in the expansion joints of tubes and tube plates in heat exchangers, welding parts including cracks or slag, overlaps of tray plates, and flange connection sealing surfaces, etc.

\subsubsection{Main Corrosion Failure Modes}

\section{Corrosion Leakage of Acid-Related Heat Exchanger Tube Bundles}

Corrosion leakage of acid-related heat exchanger is one of the common failure modes in HF acid ALK units. The tube bundle itself and the expansion joint between the tube sheet and tube bundle are the two main parts for the leakage. HF acid corrosion is the main reason. Corrosion morphology of tube bundles in acid-related heat exchangers is shown in Figure 1. 

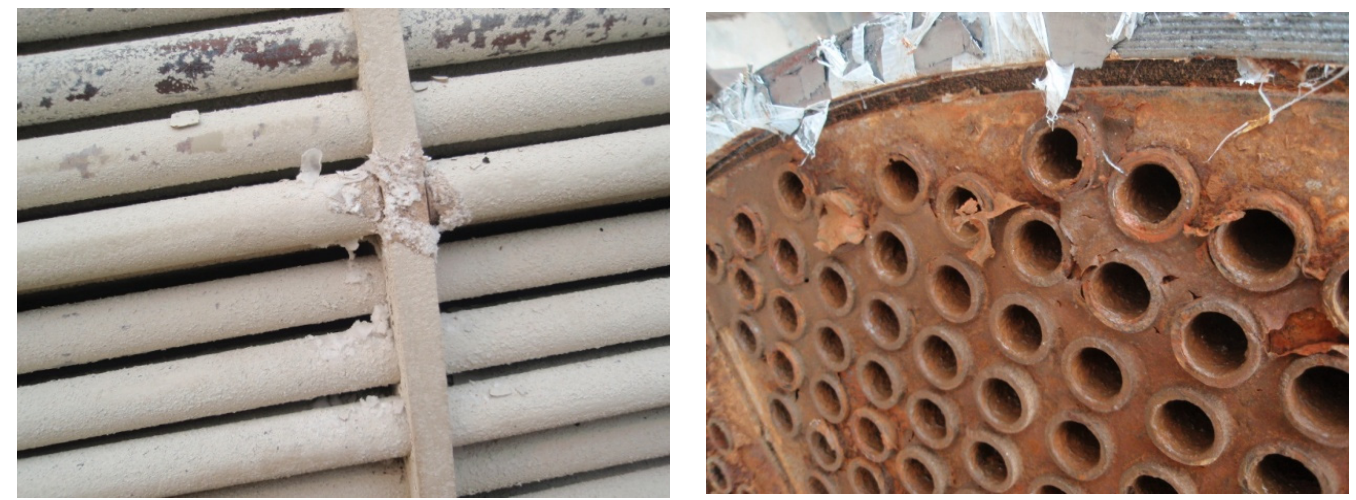

Figure 1. Corrosion morphology of acid-related heat exchanger tube bundles.

Local Metal Loss for Acid-Related Vessels and Pipelines

There is usually an even corrosion on the vessels and the pipelines under the condition of HF acid. The even corrosion produces a white corrosion product layer on the inner surfaces of the vessels or pipelines. This layer of corrosion product has a certain protective effect on the substrate. The degree of damage to the corrosion product layer varies with the operating conditions and operating environment. The corrosion rates change with the different damage degree of the corrosion product layer. As shown in Figure 2, the corrosion rate is low and the corrosion product layer is even. As shown in Figure 3, the degree of corrosion is serious and there are a large number of loose bubbles.

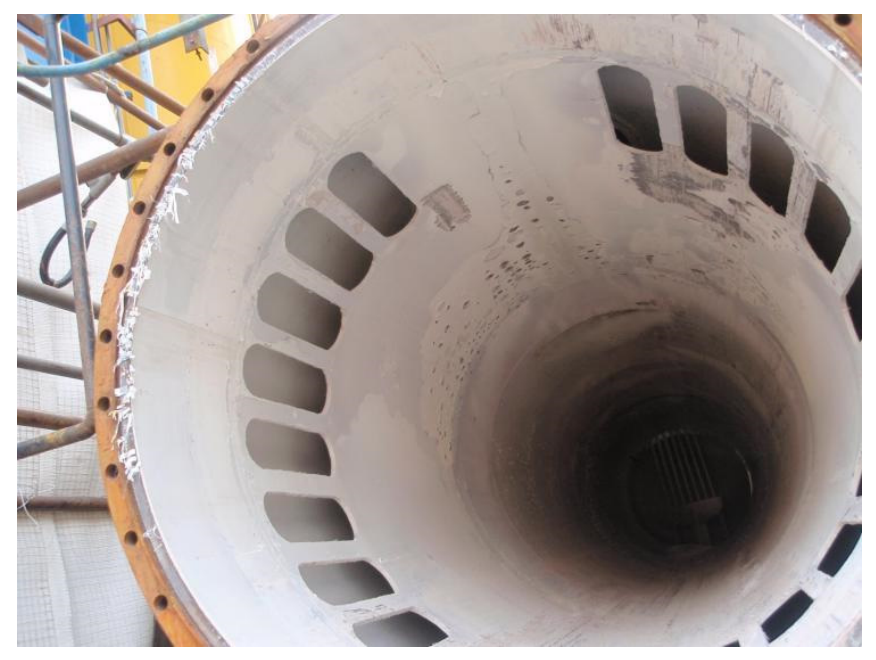

Figure 2. Even corrosion in the pipe.

\section{Corrosion and Leakage of Flange Sealing Surface}

The leakage of the flange sealing surface is one of the typical failure modes for HF acid ALK unit. The typical flange leakage morphology and gasket corrosion leakage morphology are shown in Figures 4 and 5. Subjected to the HF acid medium, the graphite composite layer of the gasket is easily peeled off. Due to the strong permeability of HF acid, the acid medium penetrates along the cracks of the graphite composite layer and contacts the metal skeleton of the gasket, which causes the sealing surface corrode. The fluoride layer can easily form in the HF acid condition. In addition, the thermal expansion coefficient of the fluoride layer is different from that of the metal, which results in further gaps in the sealing surface. Eventually, the leak will occur at flange sealing surface. 


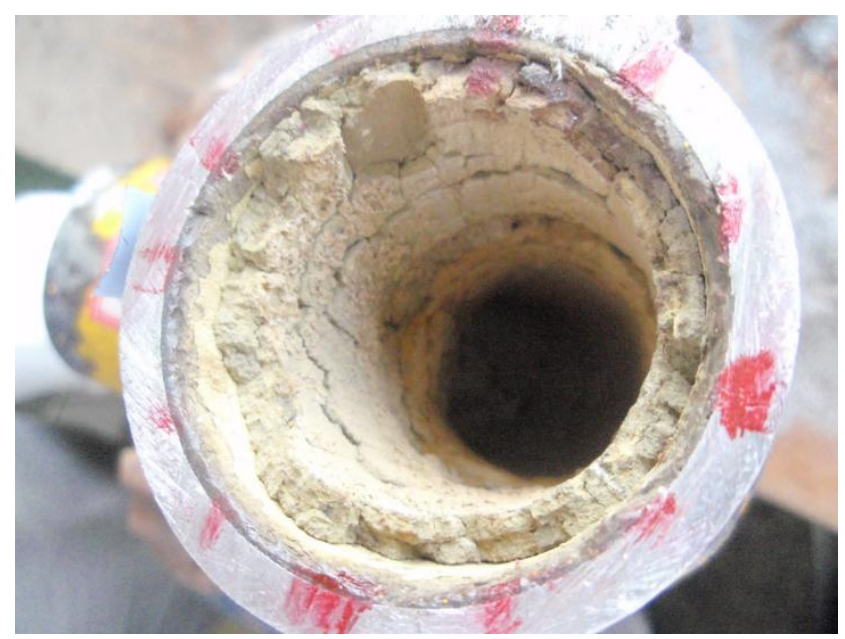

Figure 3. Serious corrosion in the pipe.

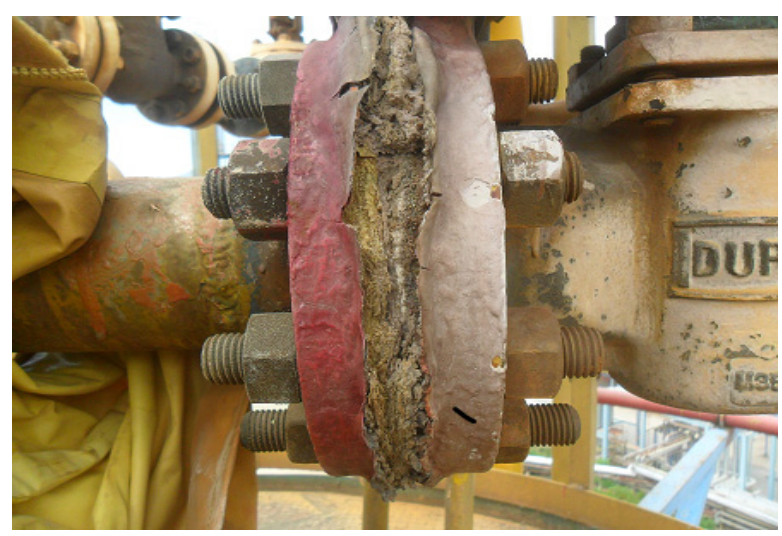

Figure 4. Typical flange leakage morphology.

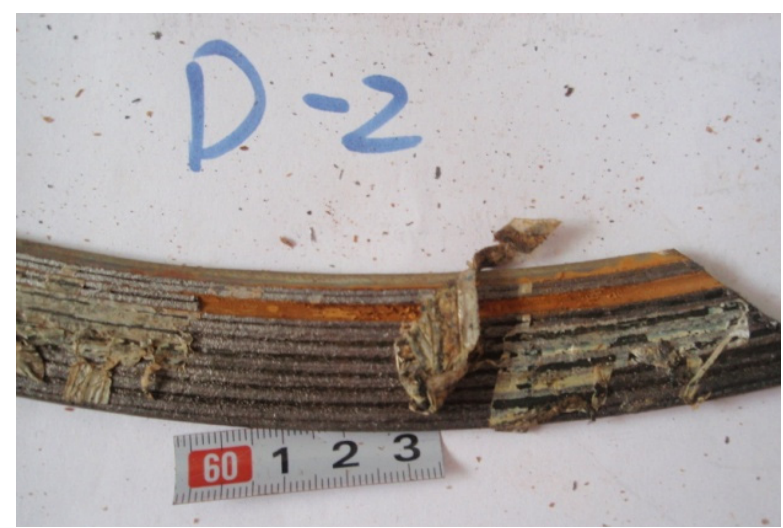

Figure 5. Typical gasket corrosion leakage morphology.

Leaking in Valve

The internal leakage of valve and safety valve is one of the common failure modes of the unit. Valves, especially safety valves, are blind ends. Acid vapor can condense at the dead corner to form a dilute acid, which corrodes the valves and causes the losses of wall thickness and internal scale layer. At the same time, the flow field change may cause the valve erosion leakage at the valve. 


\section{Corrosion Leakage of Instruments, Meters and Their Connections}

Corrosion leakage of instruments and their connections is also one of the main failure modes for static equipment in HF acid ALK unit. The instruments, meters and nozzles are blind ends, which is easy to form dilute HF acid. Another reason is that the different machining and welding process between instruments and equipment.

\section{Risk Assessments}

\subsection{Failure Possibility Calculation}

The determination of the failure possibility of the equipment is an important content of the risk assessment. The failure possibility reflects the expected failure probability of the equipment and can be calculated by the following equation:

$$
p_{f}(t)=g f f \bullet D_{f}(t) \bullet F_{M S}
$$

in which, $g f f$ is the general failure frequency of different types of equipment, which comes from the typical representative values of failure data in the petroleum refining and chemical industries. $D_{f}(t)$ and FMS are correction factors. Through calculating the correction factor, the failure probability of the vessels or pipelines can be determined finally.

\subsection{Failure Consequences Calculation}

Failure consequence is determined by the upper limit of the total amount of medium released from the equipment when the equipment fails. The larger the amount of leakage is, the more serious the potential consequences are.

\subsection{The Risk of the Equipment}

The risk of the equipment comes from the failure possibility and failure consequence. The risk calculation equation is as follows:

$$
R(t)=P_{f}(t) \cdot C(t)
$$

In which, $R(t)$ is the risk. $P_{f}(t)$ is the failure probability. $C(t)$ is the failure consequence. The risk is a function of time accumulation. With the accumulation of damage, the damage factor gradually increases. When there are multiple damage mechanisms in the equipment, the risks corresponding to each damage mechanism will be added to obtain a total timerelated risk.

\subsection{Diffusion Model under the Largest Credible Accident \\ 3.4.1. Largest Credible Accident}

The largest credible accident refers to the major accident with the most serious environmental or health hazard among all accidents whose predicted probability is not zero. Hydrofluoric acid is a dangerous chemical. Thus, there is a storage area in the factory. According to the characteristics of the process, the leakage of the hydrofluoric acid storage tank is chosen as the largest credible accident.

\subsubsection{Leak Rate Calculation}

Hydrofluoric acid leakage belongs to liquid phase leakage. The calculation of liquid leakage rate adopts Bernoulli equation, which can be obtained by the following formula:

$$
W_{n}=C_{d} \rho_{1} \frac{A_{n}}{31623} \sqrt{\frac{2000 \mathrm{~g}_{\mathrm{c}}\left(P_{\mathrm{s}}-P_{\mathrm{atm}}\right)}{\rho_{1}}}
$$

in which, $W_{n}$ is the mass leakage rate $\mathrm{kg} / \mathrm{s}, C_{d}$ is the leakage coefficient, generally equals to $0.61 . P_{S}$ is the operating pressure $\mathrm{MPa}$, and the hydrofluoric acid storage tank operating pressure is $1.12 \mathrm{MPa}$. $P_{\text {atm }}$ is the atmospheric pressure $\mathrm{MPa}$. $A_{n}$ is the leakage hole area $\mathrm{mm}^{2} . \rho_{1}$ is the density of the liquid phase medium under normal operation or storage 
conditions is $\mathrm{kg} / \mathrm{m}^{3}$, and the density of hydrofluoric acid is $1150 \mathrm{~kg} / \mathrm{m}^{3} \cdot g_{c}$ is a mechanical constant, usually $1.0(\mathrm{~kg} \cdot \mathrm{m}) /\left(\mathrm{N} \cdot \mathrm{s}^{2}\right)$ is chosen.

\subsubsection{Evaluation Standard}

The different concentration thresholds of HF harm to the human body are used as the evaluation standard, which is shown in Table 1.

Table 1. HF environmental threshold.

\begin{tabular}{ccc}
\hline Pollutants & Concentration $\mathbf{~ m g} / \mathbf{m}^{\mathbf{3}}$ & Degree of Harm to Human Body \\
\hline \multirow{3}{*}{ HF } & 400 & lethal concentration \\
& 26 & acute poisoning concentration \\
& 2 & allowable exposure concentration \\
\hline
\end{tabular}

\subsubsection{Diffusion Prediction Model}

In the current risk assessment methods, the smoke mass diffusion model is often used to simulate the diffusion process and results of toxic and hazardous substances in the atmospheric environment. To the instantaneous or short-term leakage of hydrofluoric acid storage tanks, the prediction formula is as follows:

$$
C_{r}=\frac{2 Q}{(2 \pi)^{\frac{3}{2}} \sigma_{x} \sigma_{y} \sigma_{z}} \exp \left[-\frac{\left(x-x_{0}\right)^{2}}{2 \sigma_{x}^{2}}\right] \exp \left(-\frac{y-y_{0}^{2}}{2 \sigma_{y}^{2}}\right) \exp \left(-\frac{z_{0}^{2}}{2 \sigma_{z}^{2}}\right)
$$

In which, $C_{r}$ is the concentration $\left(\mathrm{mg} / \mathrm{m}^{3}\right), u$ is the wind speed $(\mathrm{m} / \mathrm{s})$, and $Q$ is the leakage $(\mathrm{Kg}) . x, y$ and $z$ are the distance $(\mathrm{m})$ in the downwind direction, the crosswind direction and the vertical direction respectively. $x_{0}, y_{0}, z_{0}$ are the center coordinates of the smoke cluster, $x_{0}=u t, t$ is the time (s) of the leakage and diffusion. $\sigma_{x}, \sigma_{y}$ and $\sigma_{z}$ are the diffusion coefficients in downwind direction, the crosswind direction and vertical direction respectively. Usually $\sigma_{x}=\sigma_{y}$ is taken.

\section{Results and Discussions}

\subsection{Risk Results of the Equipment}

Taking the current time as the evaluation time, the risk assessment results are shown in Figure 6.
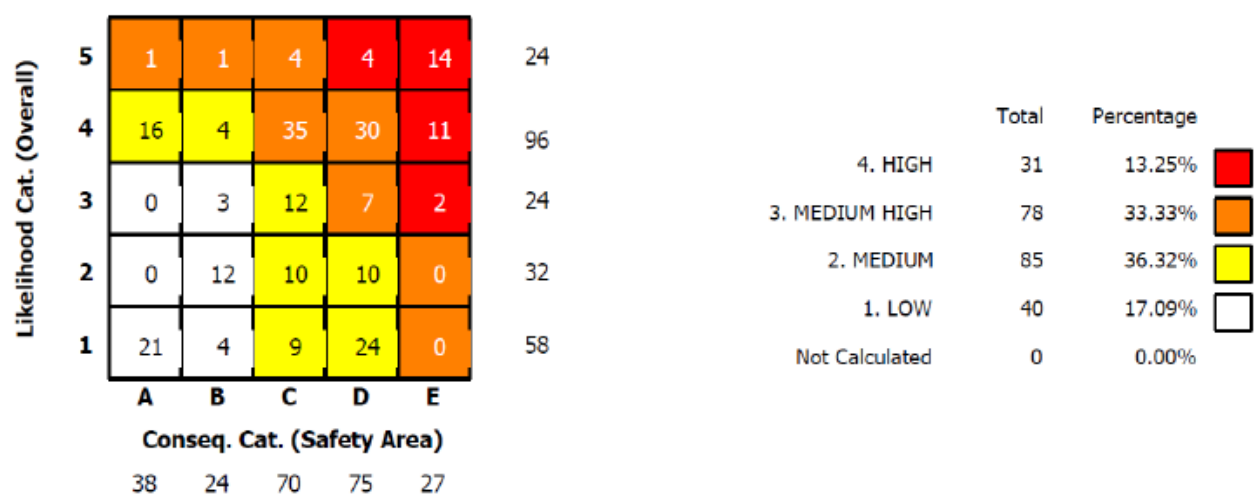

Figure 6. Risk assessment results of the equipment in ALK unit.

From the results, the overall risk of the static equipment in HF acid ALK unit is relatively high. The proportion of total risk to high risk and medium high risk is $46.58 \%$. 


\subsection{Leakage and Diffusion Results under the Largest Credible Accident}

\subsubsection{Leak Rate Calculation}

The hydrofluoric acid storage tank, the largest hazard source of the hydrofluoric acid alkylation device, was chosen as the evaluation object. The diameter of storage tank specification is $2600 \mathrm{~mm}$. The length of the storage tank is $13,400 \mathrm{~mm}$. The maximum storage capacity is 16 tons. The volume is $58 \mathrm{~m}^{3}$, and nozzle diameter of the storage tank is $50 \mathrm{~mm}$. The leakage hole area is $1952.5 \mathrm{~mm}^{2}$. According to Formula (3), the leak rate $w_{\mathrm{n}}$ is $58.65 \mathrm{~kg} / \mathrm{s}$.

\subsubsection{Prediction Results of HF Leakage and Diffusion}

The geographic coordinates of Yanshan Petrochemical are 39 north latitude and 115 east longitude. According to the statistics of the local weather station, the average wind speed for the past 20 years is $2 \mathrm{~m}$ per second. Atmospheric stability is selected D (neutral) and $\mathrm{E}$ (stable) respectively [15]. When atmospheric stability is D level, the prediction results of HF leakage and diffusion are shown in Table 2. When atmospheric stability is E level, the prediction results of HF leakage and diffusion are shown in Table 3.

Table 2. Statistical table of prediction results of HF leakage and diffusion with D level atmospheric stability).

\begin{tabular}{|c|c|c|c|c|c|c|}
\hline $\begin{array}{c}\text { Predictive Time/Min } \\
\text { Predictive Project }\end{array}$ & 5 & 10 & 15 & 20 & 25 & 30 \\
\hline Maximum landing concentration $/ \mathrm{mg} / \mathrm{m}^{3}$ & 331,985 & 57,054 & 20,367 & 9807 & 5563 & 3500 \\
\hline Corresponding distance of maximum landing concentration $/ \mathrm{m}$ & 598 & 1196 & 1795 & 2394 & 2992 & 3591 \\
\hline Radius of allowable exposure concentration range $/ \mathrm{m}$ & 723 & 1410 & 2085 & 2753 & 3416 & 4075 \\
\hline Radius of acute poisoning concentration $/ \mathrm{m}$ & 706 & 1377 & 2036 & 2688 & 3333 & 3974 \\
\hline Radius of lethal concentration /m & 687 & 1338 & 1975 & 2603 & 3224 & 3838 \\
\hline
\end{tabular}

Table 3. Statistical table of prediction results of HF leakage and diffusion with E level atmospheric stability.

\begin{tabular}{|c|c|c|c|c|c|c|}
\hline $\begin{array}{l}\text { Predictive Time/Min } \\
\text { Predictive Project }\end{array}$ & 5 & 10 & 15 & 20 & 25 & 30 \\
\hline Maximum landing concentration $/ \mathrm{mg} / \mathrm{m}^{3}$ & $1,539,023$ & 273,878 & 99,794 & 48,750 & 27,967 & 17,761 \\
\hline Corresponding distance of maximum landing concentration $/ \mathrm{m}$ & 599 & 1198 & 1798 & 2397 & 2997 & 3596 \\
\hline Radius of allowable exposure concentration range $/ \mathrm{m}$ & 683 & 1345 & 2000 & 2650 & 3297 & 3942 \\
\hline Radius of acute poisoning concentration $/ \mathrm{m}$ & 674 & 1326 & 1972 & 2613 & 3250 & 3885 \\
\hline Radius of lethal concentration/m & 663 & 1304 & 1938 & 2566 & 3191 & 3813 \\
\hline
\end{tabular}

According to the principle of maximum credible event, when the wind speed is $2 \mathrm{~m} / \mathrm{s}$ and the atmospheric stability is D level and E level, the allowable exposure concentration range radius can reach $2753 \mathrm{~m}$ and $2650 \mathrm{~m}$ respectively at $20 \mathrm{~min}$ after the accident. The nearest sensitive point, Dongfengli Community of Beijing Yanshan Petrochemical Company, is only about $2500 \mathrm{~m}$ away from the accident site. Thus, the residents of Dongfengli community must evacuate within $20 \mathrm{~min}$.

\subsection{Material Suitability Evaluation}

\subsubsection{Current Selection Analysis}

The materials of pressure equipment of the units are mainly carbon steel and Monel alloy. These pressure vessels using Monel alloy mainly include acid regeneration tower, acid cooler tube, gas stripping isobutane heater tube. Carbon steel materials for pressure vessels are 16MnDR/16MnR, A3R, 20\#/20G/20R, 25MnG, 10\#, Q235B/C. The pressure pipelines using Monel alloy mainly include regeneration ASO pipelines, the pipelines for production of HF, and circulating lye pipelines. The other pipelines basically make of 20\# steel. 
As can be seen from Figure 7, even in static anhydrous HF acid, when the temperature exceeds $66^{\circ} \mathrm{C}$, the corrosion rate already reaches $10 \mathrm{MPY}(0.254 \mathrm{~mm} / \mathrm{a})$. If $\mathrm{HF}$ acid contains a certain amount of water, the corrosion rate of HF acid will be higher when the temperature is above $66^{\circ} \mathrm{C}$ [16]. As can be seen from Figure 8, when the HF acid concentration.

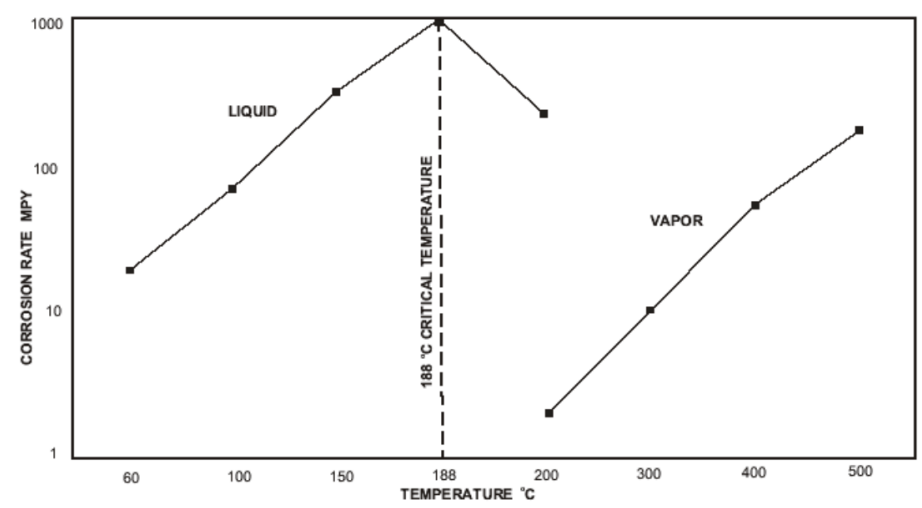

Figure 7. Curve of the corrosion rate of carbon steel as a function of temperature when immersed in static anhydrous HF for $100 \mathrm{~h}$.

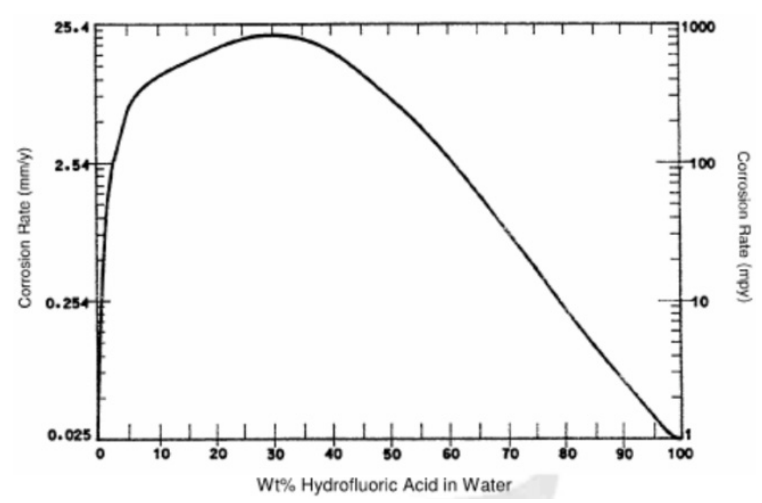

Figure 8. HF corrosion Data for Carbon Steel at 20 to $38^{\circ} \mathrm{C}$.

Is in the range of 25 to $35 \%$, the corrosion rate is close to $25.4 \mathrm{~mm} / \mathrm{a}$ [16]. As can be seen from Table 4, when the temperature is $20^{\circ} \mathrm{C}$ and the HF acid concentration is 96 to $100 \%$, the corrosion rate is only $0.03 \mathrm{~mm} / \mathrm{a}$. When the temperature is $40{ }^{\circ} \mathrm{C}$ and the $\mathrm{HF}$ acid concentration is 86 to $95 \%$, the corrosion rate is $0.21 \mathrm{~mm} / \mathrm{a}$. When the temperature is $40{ }^{\circ} \mathrm{C}$ and the $\mathrm{HF}$ acid concentration is $76-85 \%$, the corrosion rate is $0.48 \mathrm{~mm} / \mathrm{a}$ [16]. Thus, when the temperature is lower than $40{ }^{\circ} \mathrm{C}$ and the concentration is higher than $75 \%$, the carbon steel can be used. For the ALK unit, the water content of HF acid in most parts is between 1 and $3 \%$. Thus, the material can be selected from carbon steel under $66^{\circ} \mathrm{C}$.

From Table 4, it can be seen that the corrosion rate of Monel alloy in HF acid is not high in most cases. When the concentration of HF acid is between $66 \%$ and $75 \%$ and the temperature is $52{ }^{\circ} \mathrm{C}$, the highest corrosion rate approaches to $0.71 \mathrm{~mm} / \mathrm{a}$. The surface of Monel alloy 400 in HF acid has an even thinning, and the iron fluoride scale is not obvious [16]. Thus, the corrosion of Monel alloy in HF acid can be basically controlled. The main damage form of Monel alloy in HF acid is stress corrosion cracking, which mainly occurs in equipment with large residual stress, low flow rate, and high impurity.

Low alloy steel, 300 series stainless steel, and 400 series stainless steel are sensitive to HF acid corrosion and/or cracking. Thus, these materials generally should not be used in the condition with HF acid. 
Table 4. Corrosion rates of carbon steel and Monel alloy at different temperatures and concentrations.

\begin{tabular}{|c|c|c|c|c|c|c|c|c|c|c|c|c|}
\hline Material & $\begin{array}{l}\text { Concentration } / \% \\
\text { Temperature } /{ }^{\circ} \mathrm{C}\end{array}$ & $1-5$ & $6-15$ & $16-25$ & $26-35$ & $36-45$ & $46-55$ & $56-65$ & $66-75$ & $76-85$ & $86-95$ & $96-100$ \\
\hline \multirow{2}{*}{$\begin{array}{c}\text { Carbon } \\
\text { steel }\end{array}$} & 20 & / & / & 9.0 & / & / & 8.0 & 3.8 & / & / & / & 0.03 \\
\hline & 40 & 6.2 & 6.0 & 18 & 20 & 16 & 7.1 & 1.6 & 1.1 & 0.48 & 0.21 & / \\
\hline \multirow{11}{*}{$\begin{array}{l}\text { Monel } \\
\text { alloy }\end{array}$} & 20 & 0.0 & / & 0.10 & l & 0.02 & 1 & 0.0 & / & / & 0.04 & 0.05 \\
\hline & 21 & / & 0.0 & / & 0.10 & / & 0.03 & / & 0.0 & / & 0.04 & 0.05 \\
\hline & 40 & 0.18 & / & / & 0.32 & / & 0.46 & / & / & / & / & / \\
\hline & 52 & 0.25 & 0.27 & 0.38 & / & / & 0.48 & / & 0.71 & / & / & 0.05 \\
\hline & 60 & / & 0.16 & 0.25 & 0.23 & 0.30 & 0.22 & 0.06 & 0.16 & / & / & / \\
\hline & 70 & / & / & / & / & / & / & / & 0.46 & / & / & / \\
\hline & 79 & 0.42 & 0.42 & 0.06 & / & / & 0.01 & / & / & / & / & / \\
\hline & 90 & / & / & / & / & / & / & 0.16 & / & / & / & / \\
\hline & 111 & / & / & / & / & / & 0.28 & / & / & / & / & / \\
\hline & 117 & / & / & 0.25 & / & / & / & / & / & / & / & / \\
\hline & Boiling point & / & / & / & 0.02 & 0.24 & / & / & / & / & / & / \\
\hline
\end{tabular}

\subsubsection{Materials Suitability Evaluation of Key Equipment} HF Acid Storage Equipment

There are two HF acid storage tanks in the unit. The medium is more than $99 \% \mathrm{HF}$ acid. The volume is $58 \mathrm{~m}^{3}$. The risks of these two vessels are E. The two tanks have a $38{ }^{\circ} \mathrm{C}$ operating temperature and a $1.12 \mathrm{MPa}$ operating pressure. The materials are $\mathrm{A} 3 \mathrm{R}$ and $20 \mathrm{R}$. From the above analysis, it can be seen that at $38^{\circ} \mathrm{C}$, carbon steel has a low corrosion rate in a $99 \% \mathrm{HF}$ acid environment. Therefore, it is appropriate to use carbon steel for the two acid-related storage tanks. The operation files of the two tanks verified that the shells have not been corroded. Only some valves have experienced internal leakage. Attention should be paid to the corrosion of valves.

\section{Reaction and Regeneration Section}

Acid cooler, acid sedimentation tank, and acid re-contactor are used in the reaction and regeneration section with operating temperature lower than $66^{\circ} \mathrm{C}$. The risks of these three vessels are E. Under normal operating conditions, HF acid is about $80 \%$. The water content in HF acid is within 3\%. Therefore, carbon steel can be used for these vessels. Due to a certain amount of water in the medium, low concentration HF acid may be formed in local area.

Acid regeneration tower feed vaporizer, and acid regeneration tower are used in the reaction and regeneration section with operating temperature higher than $66{ }^{\circ} \mathrm{C}$. Both vessels use Monel alloys with high operating temperatures. The material grades are matched. But, some attention should be paid to problems such as corrosion and cracking of the tube bundle, the connection between the tube bundle and the tube sheet, and leakage of the flange sealing surface.

\section{ASO Oil Treatments Section}

The HF content in the ASO oil discharged from the bottom of the acid regeneration tower is about $3 \%$. The temperature is more than $100{ }^{\circ} \mathrm{C}$. The corrosion of these pipelines should be serious. The ASO oil can only be discharged after being neutralized by alkali. ASO oil has a high failure consequence before alkali neutralization. Thus, several ASO oil pipelines before the alkali neutralization, including acid-soluble oil mixers, all make of Monel alloy. After alkali neutralization, the risk of equipment is not high. Therefore, acid-soluble oil-alkali washing tank and acid-soluble oil storage tank all use carbon steel.

\section{Fractionation Column Top Section}

The main equipment in the tower top circuit mainly includes stripped isobutane heater, cycle isobutane cooler, fractionation tower feed cycle butane heat exchanger, main feed of fractionation tower-AKL oil heat exchanger, main fractionation tower- condensation tower, 
main fractionation tower, propane stripper towerand main fractionation tower-Stripping tower top reflux tank.

From the equipment operation records, it can be seen that the relevant connecting flanges and valves on the upper part of the main fractionation tower, the short pipe connected to accessory equipment occurred leaking many times. The tower trays have been seriously corroded. HF acid with a low concentration can be formed on the upper part of the tower, which has a high corrosion and a high failure consequence.

The materials of upper parts of the main fractionation tower, the propane stripper tower, the main fractionation tower-Stripping tower top reflux tank, and the main fractionation tower- condensation tower are carbon steel. Considering the balance of economy and safety, Monel-lined materials are suggested to use on the acid-related parts of these vessels. At the same time, Monel alloys are suggested to use on their auxiliary nozzles. Although the cost will increase, the corrosion rate and damage sensitivity will also be greatly reduced.

Propane and Butane Defluorination Section

The butane from the middle of the main fractionation tower and the propane from the bottom of the propane stripping tower both contain small amount of HF acid. Propane and butane form free water during defluorination. The remaining small amounts of HF acid are dissolved in water, which may cause local HF acid corrosion. The amount of hydrofluoric acid is small, large scale corrosion damage will not happen. Therefore, the material selection of the equipment in this section is suitable.

\section{Material Suitability Evaluation of Other Equipment Materials}

Other equipment in the corrosion circuit does not involve in HF acid. There is no clear corrosion mechanism. The overall temperature is not high. Thus, the use of carbon steel is suitable.

\subsection{Management Strategy}

\subsubsection{Process Anti-Corrosion}

The water content of HF acid has a big influence on the corrosion rate of HF acid. Thus, the water content in the equipment of the raw materials, dehydrated raw materials, circulating acid, top of fractionation tower, circulating isobutane, butane and propane after defluorination should be checked periodically. The water content is controlled in an allowable range. In terms of process monitoring, the $\mathrm{PH}$ value, impurity content and acid content of the circulating water should be monitored in order to find in time whether HF acid leaks into the circulating water through the heat exchanger tube bundle. If a leaking is found, the corresponding heat exchanger should be switched down and be repaired timely. At the same time, the corrosion of circulating water containing HF acid on other equipment should be avoided.

\subsubsection{Equipment Anti-Corrosion}

When repairing or replacing equipment of the HF acid ALK unit, the following aspects should be paid attention to:

(1) To control the residual elements of carbon steel welds, such as chromium, nickel, copper, it is better to use hydrogen-resistant cracking steel and welding materials.

(2) To prevent hydrogen embrittlement of carbon steel and stress corrosion cracking of Monel alloy, stress relief heat treatment should be performed. A stress relief heat treatment should also be conducted for the elbow of U-shaped tube heat exchanger.

(3) The hardness should be tested for new vessels, new pipelines or repaired welds before being put into use. The high hardness equipment is avoid to put into service. 


\section{Conclusions}

(1) The overall risk of the static equipment in HF acid ALK unit is relatively high. The proportion of total risk to high risk and medium high risk is $46.58 \%$. The HF acid has a high corrosion with a medium HF acid concentration. The flange sealing surfaces are easy to corrode due to HF acid condensation or aggregation.

(2) According to the principle of maximum credible event, when the wind speed is $2 \mathrm{~m} / \mathrm{s}$ and the atmospheric stability is D level and E level, the allowable exposure concentration range radius can reach $2753 \mathrm{~m}$ and $2650 \mathrm{~m}$ respectively at $20 \mathrm{~min}$ after the accident. The nearest sensitive point, Dongfengli Community of Beijing Yanshan Petrochemical Company, is only about $2500 \mathrm{~m}$ away from the accident site. Thus, the residents of Dongfengli community must evacuate within $20 \mathrm{~min}$.

(3) The overall failure risk is high for vessels and pipelines with carbon steel. If a large number of carbon steel materials are upgraded to Monel, the cost is high. A regular replacement method can be used to maintain the reliability of the equipment. The management strategies of process anti-corrosion and equipment anti-corrosion of the ALK unit were proposed. It is meaningful for reducing the number of shutdowns of ALK units and maintaining safe and stable operation of the unit.

Author Contributions: Conceptualization, W.W. and D.L.; methodology, W.W. and D.L.; software, X.L.; validation, X.L, L.L. and D.L.; formal analysis, L.L.; investigation, X.L.; re-sources, D.L.; data curation, L.L.; writing - original draft preparation, W.W; writing-review and editing, X.L.; visualization, L.L.; supervision, D.L.; project administration, D.L.; funding acquisition, D.L. All authors have read and agreed to the published version of the manuscript.

Funding: This research is supported by Research and development of key technology and equipment for typical petrochemical process safety assurance (2018YFC0808600), and the research special project of public welfare industry (201210022).

Institutional Review Board Statement: Not applicable.

Informed Consent Statement: Not applicable.

Data Availability Statement: Not applicable.

Conflicts of Interest: The authors declare no conflict of interest.

\section{References}

1. Philippa, M.; John, W. Extending the internal examination intervals for pressure vessels using a Risk Based Inspection (RBI) approach. Weld. Cut. 2010, 9, 160-166.

2. Choi, S.-C. The Implementation of Risk-Based Inspection for the Refinery Plant. Corros. Sci. Technol. 2012, 11, 43-47. [CrossRef]

3. Wu, W.-F.; Lin, S.-R.; You, J.-S. Risk-based inspection and maintenance in process plants and their practices in Taiwan. J. Chin. Inst. Eng. 2016, 39, 392-403. [CrossRef]

4. Akanni, J.; Alonge, O. Effective implementation of Risk Based Inspection (RBI) approach in asset integrity man-agement of oil and gas facilities. In Proceedings of the ASME 2015 Pressure Vessels and Piping Conference, Paris, France, $19-23$ July 2015.

5. Whittle, D.K.; Lorenzo, D.K.; Kirkman, J.Q. Results of alkylation unit hazard, operability study are analyzed and summarized. Oil Gas J. 1989, 87. Available online: https:/ / www.osti.gov/biblio/5718837 (accessed on 19 August 2021).

6. Gary, B.; Teddy, B.; Kristin, B.-B.; Dauber, J.; Frederick, J.; Hemingway, D.; Lefton, J.; Lidston, J.; Lewis, T.; Lippin, T.; et al. A Risk too Great. Hydrofluoric Acid in U.S. Refineries. Available online: http://assets.usw.org/resources/hse/pdf/A-Risk-Too-Great. pdf (accessed on 12 March 2013).

7. Muralidhar, R.; Jersey, G.; Krambeck, F.; Sundaresan, S. A two-phase release model for quantifying risk reduction for modified HF alkylation catalysts. J. Hazard. Mater. 1995, 44, 141-183. [CrossRef]

8. Juliana, P.; Gómez, S.; Ruiz, I. Application of HAZOP, LOPA and SIL to an Alkylation Unit in a Refinery: A Case Study. Chem. Eng. Trans. 2020, 82, 343-348.

9. Wang, R.; Wanling, Z.; Weihua, W. Risk Assessment (RBI) and Inspection and Verification on Catalytic Cracking Unit. Sci. Technol. Eng. 2011, 11, 7091.

10. Yan, T.; Yang, P.; Chen, W. Application of RBI technology in delayed coking unit of refinery. Chem. Mach. 2011, 38 , 226-227, 235.

11. Wang, B. Application of RBI in ethylene plant. Chem. Mach. 2012, 39, 512-514, 535.

12. Shi, K.; Jiang, H.; Zhao, W.; Lu, D. Risk analysis of pipeline system of natural gas installation based on RBI. Petrochem. Safety Environ. Prot. Technol. 2014, 31, 37-39. 
13. Liang, J.; Li, Z.; Li, Y.; Li, H. Application of risk-based inspection (RBI) in pressure-bearing special equipment at LNG receiving station. Pet. Chem. Equip. 2018, 43, 75-79.

14. Liu, W.; Sun, J.; Ru, W. Risk assessment of pressure vessel for methanol gasification unit based on RBI technology. Nondestruct. Test. 2019, 36, 23-26.

15. American Institute of Chemical Engineers. Guidelines for Use of Vapor Cloud Dispersion Models; American Institute of Chemical Engineers: New York, NY, USA, 1996.

16. NACE International. NACE Publication 5A171 (2001 Edition), Materials for Storing and Handling Commercial Grades of Aqueous Hydrofluoric Acid and Anhydrous Hydrogen Fluoride; NANCE: Huston, TX, USA, 2007. 